Proc. 13th International School on Theoretical Physics: Symmetry and Structural Properties of Condensed Matter

\title{
Increase of implant temperature during laser disinfection
}

\author{
L. PYZIAK ${ }^{a, *}$, T. WiĘCEK ${ }^{a}$, D. PALIGA ${ }^{b}$ AND R. MAKSYMOWICZ ${ }^{c}$ \\ ${ }^{a}$ Rzeszów University of Technology, al. Powstańców 12, 35-959 Rzeszów, Poland \\ ${ }^{b}$ Dental office Renata and Dariusz Paliga s.c., al. Niepodległości 3/2, Rzeszów, Poland \\ c"DentAR" s.c. Agata and Radosław Maksymowicz, A. Mickiewicza 21, Rzeszów, Poland
}

\begin{abstract}
Peri-implantitis is the destructive inflammatory process affecting both the soft and the hard tissues, which surround dental implants. In the worst case, it may lead to a tooth or an implant loss. To stop this process, bacteria have to be killed. For this purpose, the photo activated disinfection method can be used. The aim of our research is to examine, if the implementation of the photo activated disinfection method may cause an increase of the temperature and damage the surrounding tissues. A periodontal pocket model has been made. The research has involved the use of a diode $400 \mathrm{~mW}$ laser with a wavelength of $635 \mathrm{~nm}$ and toluidine blue. The recorded increases in the temperature have proved that such a type of disinfection is safe for tissues surrounding the implant.
\end{abstract}

DOI: 10.12693/APhysPolA.135.1265

PACS/topics: 42.62.Be, 87.50.W-, 87.19.xb

\section{Introduction}

Periodontal diseases affect around $30 \%$ of the adult population. The periodontal pocket is a disease manifesting in an appearance of a space below the gum tissue alongside a tooth. The depth of this space can reach $3-10 \mathrm{~mm}$. The presence of deep periodontal pockets (greater than $6 \mathrm{~mm}$ ) varies from $10 \%$ to $15 \%$ in adult populations [1]. Bacteria (Porphyromonas gingivalis, Actinobacillus actinomycetemcomitans) penetrate into this space and cause inflammation of periodontal tissues and resorption of the alveolar bone. It causes further weakening of the soft and bone tissue that keeps the teeth. As a result a tooth can be lost. This process is called peri-implantitis. In the classical therapy, the process of reduction of the pathogen bacteria is done by mechanical debridement together with an antimicrobial treatment. The complete elimination of bacteria is very difficult with this method [2], therefore other procedures had to be found. The first information on an application of antimicrobial photodynamic therapy, consisting of application of a laser, photosensitizer, and oxygen, was presented in 2006 [3]. Photo activated disinfection has been introduced in periodontology as an aid for disinfection of root canals [4] and periodontal pockets [5-8].

It is the laser therapy in which the laser energy itself is not particularly lethal to bacteria, but is used to achieve photochemical activation of photosensitizer. The photosensitizer releases singlet oxygen which causes bacteria membrane and DNA damage. Toluidine blue is widely used in a dental practice. In medical concentrations, such a formulation is not toxic, does not stain tissues, does not require the use of high-power lasers, and is relatively cheap. Most photosensitizers are activated by red light between 630 and $700 \mathrm{~nm}$ [9]. The side effect of using laser

*corresponding author; e-mail: 1.pyziak@prz.edu.pl light is an increase of temperature that can be dangerous for healthy neighbouring tissues. Periodontal tissues are not damaged if the temperature increase is kept below $5^{\circ} \mathrm{C}$. A threshold temperature increase of $7^{\circ} \mathrm{C}$ is commonly considered as the highest thermal change, which is biologically acceptable to avoid periodontal damage [10]. There are several papers (e.g. [7, 11, 12]), where authors have presented results concerning the temperature increase caused by PAD procedure. In [7] real extracted human teeth has been used. In their test, the authors have used a laser at $660 \mathrm{~nm}$, where, during $60 \mathrm{~s}$ interval the output power was $20 \mathrm{~mW}$ (power density $0.09 \mathrm{~W} / \mathrm{cm}^{2}$ and energy density $5.4 \mathrm{~J} / \mathrm{cm}^{2}$ ).

In our studies, similar measurements have been made for artificial implants, which can be mounted in the place of lost teeth. A $635 \mathrm{~nm}$ laser with power $400 \mathrm{~mW}$ and time of irradiation of $30 \mathrm{~s}$ has been used. Due to the use of a more powerful laser, the disinfection process is more efficient as the laser light penetrates deeper into the tissue. As a photosensitizer we have applied toluidine blue.

\section{Sample preparation}

In our experiments two different implants have been used. Their parameters and appearance are presented in Table I and Fig. 1, respectively. Both implants are made of titanium alloys and they differ in size and weight.

To create the periodontal pocket there has been used the wax, which has been glued to the implant. In the next step, the implant has been inserted in a thermally conductive silicone. The thermal conductivity of this silicone $(1.2 \mathrm{~W} /(\mathrm{m} \mathrm{K}))$ is similar to the thermal conductivity of the tissues of the human body (0.837$2.093 \mathrm{~W} /(\mathrm{m} \mathrm{K}))[7]$.

After the silicone has congealed, the wax has been melted with hot water to form a pocket. The implant fixed in the silicone prepared for the experiment is shown in Fig. 1b. 
Implants and their parameters

TABLE I

\begin{tabular}{c|l|c|c|c}
\hline \hline & Manufacturer & {$[\mathrm{mm}]$} & $L[\mathrm{~mm}]$ & $m[\mathrm{~g}]$ \\
\hline 1 & SGS dental implant & 4.2 & 10 & 0.2831 \\
2 & octagon implant & 4.1 & 12 & 0.5102
\end{tabular}
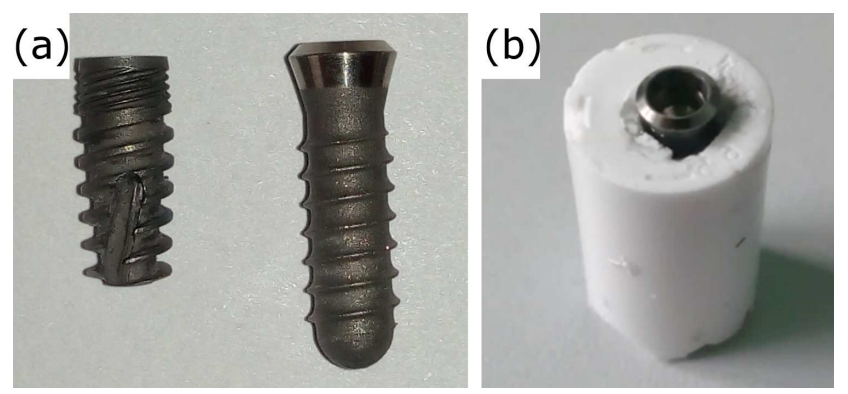

Fig. 1. Implants: (a) SGS dental on the left (1 in Table I) and octagon on the right (2 in Table I); (b) the implant prepared to measurements.

\section{Measurement procedure}

The implant prepared in such a way has been placed in a water bath with a constant temperature of $36.6^{\circ} \mathrm{C}$. The pocket has been filled with a photosensitizer (toluidine blue). For temperature measurements three K type thermocouples with a Picolog TC08 temperature recording device have been used. The accuracy of these measurements was $0.01{ }^{\circ} \mathrm{C}$. The first thermocouple has been placed in a pocket filled with the photosensitizer. The second one has been attached to the implant, and the last one has been placed in the water bath. Diagrams showing the experimental setup and implant prepared for measurements as well as thermocouples with the end of the optical fiber placed in the periodontal pocket are shown in Fig. 2 and Fig. 3, respectively (please note that Fig. 3 is the magnified fragment of Fig. 2).

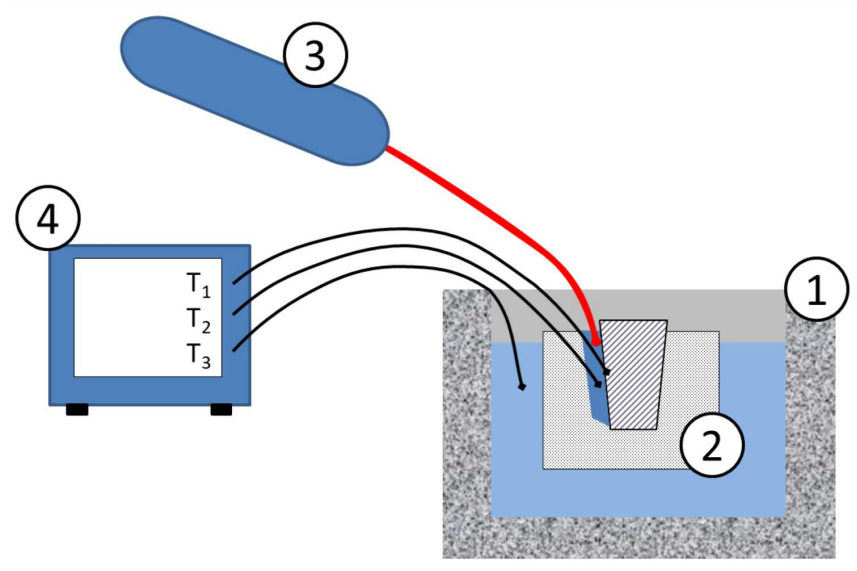

Fig. 2. Experimental setup: 1 - water bath, 2- sample, 3 - laser head with optical fiber, 4 - temperature recording device with thermocouples.

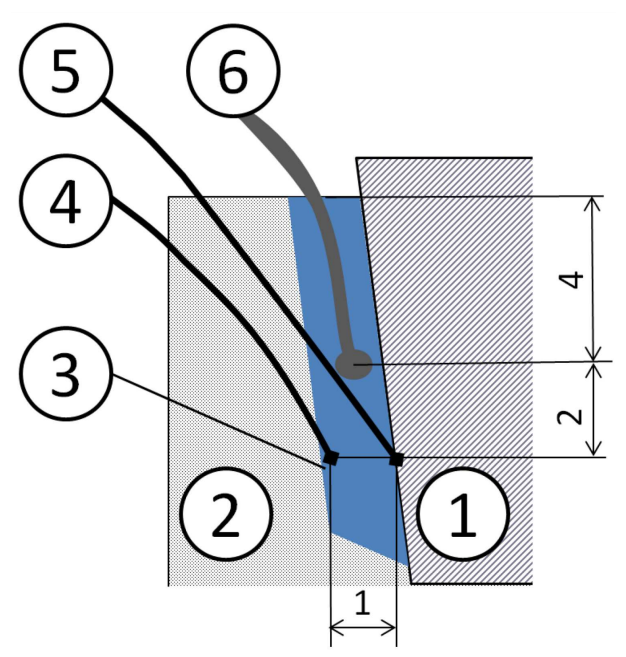

Fig. 3. Sample with connected thermocouples and fiber-optic cable: 1 - implant, 2 - thermally conductive silicone, 3 - periodontal pocket filled with toluidine blue, 4 - thermocouple for measurements of photosensitizer temperature, 5 - thermocouple for implant temperature measurements, $6-$ optical fiber. All dimensions are given in $\mathrm{mm}$.

In the prepared periodontal pocket model, the volume of toluidine blue was about $28 \mathrm{~mm}^{3}$. Such a result is comparable with the volume used in real medical treatment. To activate reaction in the photosensitizer, diode continuous laser of $400 \mathrm{~mW}$ with the wavelength of $635 \mathrm{~nm}$ has been used (Lasotronix Laser DiodeLXmodel SmartSPro). Time of lighting has been set for $30 \mathrm{~s}$. Due to such a choice of parameters, power density of $3.5 \mathrm{~W} / \mathrm{cm}^{2}$ and energy density of $105 \mathrm{~J} / \mathrm{cm}^{2}$ have been achieved. The laser light has been inserted into the pocket with a fiberoptics cable ended with a specialized tip (universal PACT Light Guide, Cumdente GmbH (Fig. 4)). The construction of this tip causes emission of the light directly as well as on the side walls (Fig. 4).

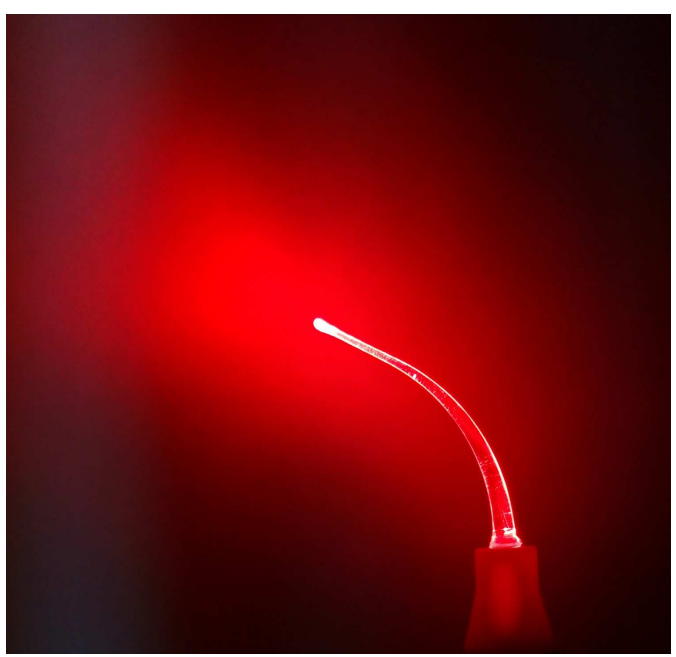

Fig. 4. Universal PACT light guide, Cumdente GmbH. 


\section{Results}

For each implant 20 measurements of temporal temperature change of the toluidine blue, implant, and environment have been carried out. An exemplary result of temperature measurement as a dependence of time is shown in Fig. 5. The temperature recording in all measurements has been started a few seconds before the laser was switched on. The laser action lasted for $30 \mathrm{~s}$. The graph in Fig. 5 shows a sharp increase in the temperature of both the photosensitizer filling of the pocket and the implant. The values of these temperature increases, has been determined by the measuring device and saved to the computer's memory. Mean values and standard deviations of the values of growth of photosensitizer and implant temperature for each tested implant have been calculated. The obtained values are presented in Fig. 6.

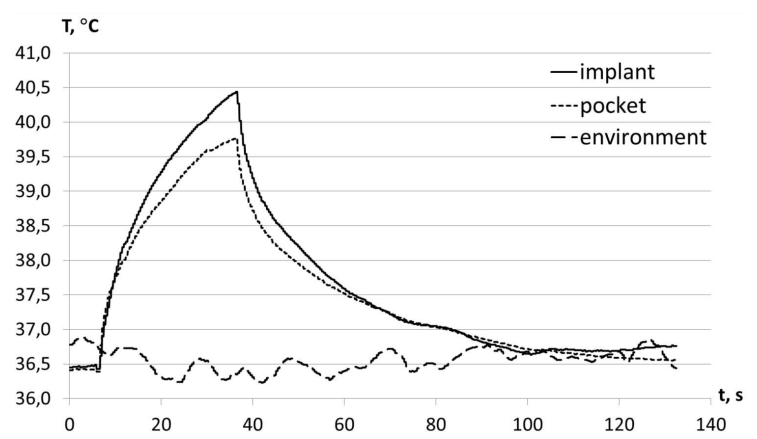

Fig. 5. Dependence of temperature of implant (solid line), pocket (dotted line) and environment (dashed line) on time.

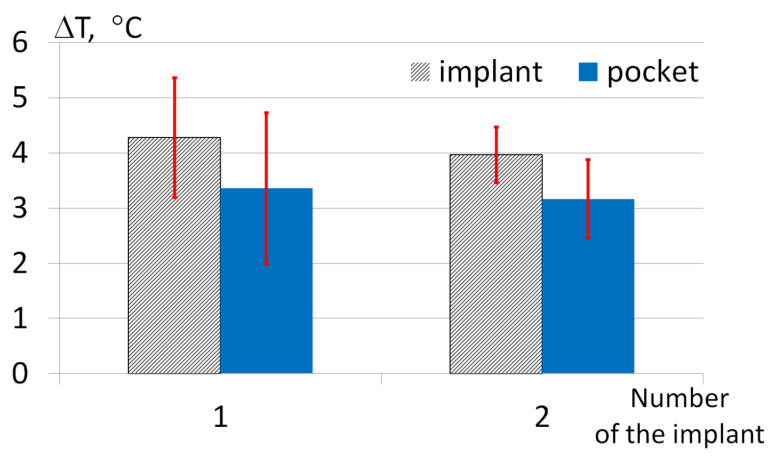

Fig. 6. Mean values and standard deviations of the values of growth of photosensitizer and implant temperature (implants were described in Table I).

\section{Conclusions}

As a result of our research an increase of the temperature, both the photosensitizer and the implant, under the influence of laser light has been observed. Higher temperatures have been, however, achieved by the implant. The temperature of the second implant has been lower due to its higher mass.

Increase in temperature in both cases is smaller than $4.3^{\circ} \mathrm{C}$. It can be concluded that the temperature rise achieved during PAD treatment is not dangerous for tissues surrounding the implant. Therefore PAD is a safe procedure for the treatment of peri-implantitis, where instead of a tooth in the gum an implant can be inserted.

\section{References}

[1] P.E. Petersen, H. Ogawa, Periodontology 2000 60, 15 (2012).

[2] E.S. Chaves, M.K. Jeffcoat, C.C. Ryerson, B. Snyder, J. Clin. Periodontol. 27, 897 (2000).

[3] T. Maisch, Lasers Med. Sci. 22, 83 (2007).

[4] S.J. Bonsor, R. Nichol, T.M.S. Reid, G.J. Pearson, Brit. Dent. J. 201, 101 (2006).

[5] A.A. Takasaki, A. Aoki, K. Mizutani, F. Schwarz, A. Sculean, Chen-Ying Wang, G. Koshy, G. Romanos, I. Ishikawa, Y. Izumi, Periodontology 2000 51, 109 (2009).

[6] A.M. Maria, I.G. Ursarescu, S. Solomon, L. Foia, Balkan J. Dent. Med. 20, 29 (2016).

[7] H. El Yazami, T. Zeinoun, S. Bou Saba, L. Lamard, A. Peremans, M. Limme, S. Geerts, M. Lamy, S. Nammour, Lasers Med. Sci. 25, 655 (2010).

[8] R. Smeets, A. Henningsen, O. Jung, M. Heiland, Ch. Hammächer, J.M. Stein, Head Face Med. 10, 34 (2014).

[9] N. Komerik, A.J. MacRobert, J. Environ. Pathol. Toxicol. Oncol. 25, 487 (2006).

[10] P.J. Piccione, Dent. Clin. North Am. 48, 795 (2004).

[11] C.M. Cobb, J. Periodontol. 77, 545 (2006).

[12] B. Dickers, L. Lamard, A. Peremans, S. Geerts, M. Lamy, M. Limme, E. Rompen, R.J.G. De Moor, P. Mahler, J.P. Rocca, S. Nammour, Lasers Med. Sci. 24, 81 (2009). 